\title{
Four Consecutive Recurrent Abortions in Patient with Essential Thrombocythemia
}

\author{
Hui-Gyeong Seo, Hye-Na Park, Hye-Ji Jeon, Yun-Sook Kim \\ Department of Obstetrics and Gynecology, Soonchunhyang University Cheonan Hospital, Soonchunhyang University College of Medicine, Cheonan, Korea
}

\begin{abstract}
Essential thrombocythemia (ET) is characterized by most cases in which platelet counts exceed 1 million/ $\mu \mathrm{L}$. ET is usually no symptoms during non-pregnancy, but arterial and venous thrombosis and hemorrhage may develop in pregnancy. Pregnancy in these patients is associated with many complications in both pregnant women and fetuses such as recurrent abortion, intrauterine fetal growth restriction, preterm delivery, preeclampsia, and stillbirth. In these patients, aspirin, low-molecular-weight heparin (LMWH), and interferon alpha (INF-a) are recommended during pregnancy. We report a case of four consecutive abortions despite being treated with INF-a, low dose aspirin, and LMWH in patient with ET.
\end{abstract}

Keywords: Essential thrombocythemia; Recurrent abortion; Interferon-alpha; Aspirin

\section{INTRODUCTION}

Essential thrombocythemia (ET) is a chronic disorder involving multipotent hematopoietic progenitor cell manifested clinically by excessive production of platelets. Pregnancy associated ET is divided into essential and reactive ET, which is caused by infection, inflammation, iron deficiency anemia, malignancy, and some sorts of drugs [1]. ET is a rare disorder, with an incidence of 0.52.5/100,000 individuals and a markedly female predominance [2]. The most common pregnancy-related complication in patients with ET is spontaneous abortion during the first trimester. Placental micro-infarctions due to increased platelet number and placental damage from autoantibodies are among the underlying pathological basis of first trimester miscarriage [3]. Therapy of patients with ET during pregnancy is a controversial issue. The therapeutic methods that can be selected include no treatment or administration of cytoreductive or antiplatelet agents. However, cytoreductive drugs have not been recommended for use during pregnancy, particularly during the first trimester of pregnancy [4]. Anagrelide is also not advised during pregnancy because the efficacy of this drug for the fetuses has not been adequately studied
[5]. Interferon alpha (INF- $\alpha$ ) is a selectable drug of choice in early pregnancy [6].

\section{CASE REPORT}

A 40-year-old woman with a history of two normal births and three consecutive abortions visited Soonchunhyang University Cheonan Hospital with a threatened abortion with vaginal bleeding at 9 weeks of pregnancy.

She was diagnosed with ET by chance in a general blood test in 2005. She had no Janus kinase 2 (JAK2) V617F mutation. The platelet count at the time of diagnosis was $600 / \mathrm{mm}^{3}$ and did not receive any treatment until the first cesarean section in 2006. After the first child, she took $100 \mathrm{mg}$ aspirin and $1 \mathrm{mg}$ anagrelide until the time of second pregnancy, and the average platelet count at that time was $750 / \mathrm{mm}^{3}$. During the second pregnancy, she took $100 \mathrm{mg}$ aspirin only and she had a second cesarean section in 2010. Platelet count at the time of the second child was $1,663 / \mathrm{mm}^{3}$. Low-molecular-weight heparin (LMWH) was used to prevent thrombosis before surgery, on the day of surgery, and 1 day after surgery. Since then, she had taken $100 \mathrm{mg}$ aspirin only, and the
Correspondence to: Yun-Sook Kim

Department of Obstetrics and Gynecology, Soonchunhyang University Cheonan Hospital,

31 Suncheonhyang 6-gil, Dongnam-gu, Cheonan 31151, Korea

Tel: +82-41-570-2150, Fax: +82-41-571-7887, E-mail: drsook@schmc.ac.kr

Received: Jan. 13, 2017 / Accepted after revision: Feb. 15, 2017
(C) 2017 Soonchunhyang Medical Research Institute This is an Open Access article distributed under the terms of the Creative Commons Attribution Non-Commercial License (http://creativecommons.org/licenses/by-nc/4.0/). 
platelet count was $1,862 / \mathrm{mm}^{3}$, so she had a bone marrow biopsy. Bone marrow biopsy specimen showed mainly proliferation of the megakaryocytic lineage with increased numbers of enlarged and mature megakaryocytes, which were appropriate for ET (Fig. 1). She has not been followed for two years until 2013. According to the patient, she was not treated for two years and had two repeated spontaneous abortions at the beginning of pregnancy. Platelet count at that time was $1,343 / \mathrm{mm}^{3}$. She has been treated with anagrelide from September 2013 through July 2014 because she no longer wants to get pregnant. After that, however, she wanted to get pregnant again, stopped taking anagrelide and received an INF- $\alpha 5.0 \times 10^{6}$ IU subcutaneous injection five times a week until 2015. She had severe headache and muscle pain when INF- $\alpha$ was injected. So she took $650 \mathrm{mg}$ acetaminophen together. Again, her baby was aborted three consecutive times in the ninth week of

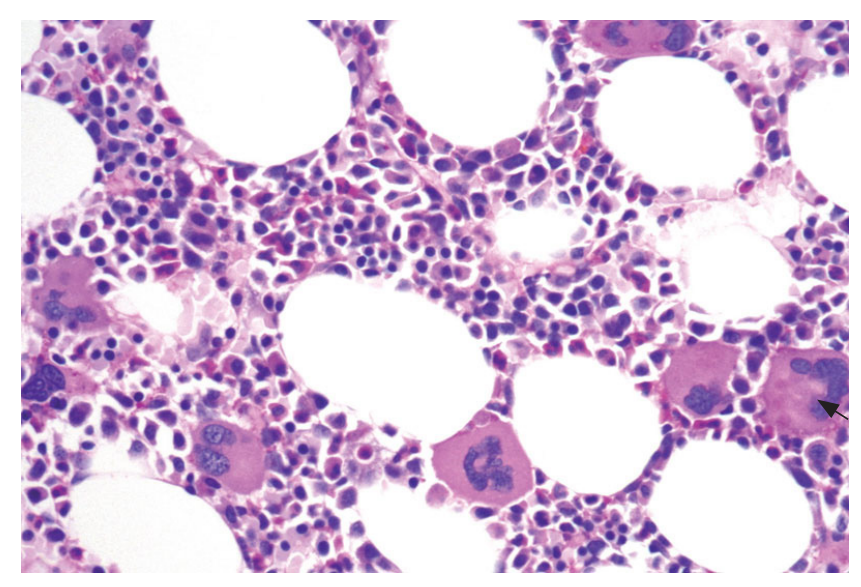

Fig. 1. Bone marrow biopsy specimen showed mainly proliferation of the megakaryocytic lineage with increased numbers of enlarged and mature megakaryocytes. They reveal abundant amounts of mature cytoplasm and deeply lobulated and hyperlobulated (stag-horn-like) nuclei (arrow) (PAS, H\&E, × 400). pregnancy. She wanted more pregnancy again after termination. The headache was so severe that the dose of INF- $\alpha$ was halved. We also planned to add LMWH. As soon as she got pregnant she took $100 \mathrm{mg}$ aspirin and was given an INF- $\alpha 2.5 \times 10^{6} \mathrm{IU}$, enoxaparin 6,000 IU injection every day until she was hospitalized (Table 1).

The patient had no notable family history. On physical examination, she had multiple bruises on her both arms, and her vital signs were normal. Her weight was $80 \mathrm{~kg}$, her blood pressure was 130/80 $\mathrm{mm} \mathrm{Hg}$, her pulse rate was 65 beats/min, her respiratory rate was 20 breaths $/ \mathrm{min}$, and her body temperature was $37^{\circ} \mathrm{C}$. The patient also showed hemoglobin was $11.6 \mathrm{~g} / \mathrm{dL}$ (range, 12.0 to $16.0 \mathrm{~g} / \mathrm{dL}$ ), white blood cell count $10,880 / \mu \mathrm{L}$ (range, 4,000 to $10,000 / \mu \mathrm{L}$ ), platelet $1,048 / \mathrm{mm}^{3}$ (range, 130 to $400 / \mathrm{mm}^{3}$ ), and coagulation profiles were within normal limits. The patient was negative for hepatitis B, syphilis, and human immunodeficiency viruses. The patient also showed HLA B51 (-), anti-neutrophil cytoplasmic antibody (-), von Willebrand antigen 54.4 (range, 47 to 197), and leucine aminopeptidase score 70 (range, 30 to 130).

After 1 week of hospitalization, vaginal spotting was stopped and she was discharged and visited the outpatient clinic for 13 weeks of pregnancy. Unfortunately, there was no fetal heartbeat on the ultrasound. But the addition of aspirin and LMWH extended the abortion period by five weeks. The fetus was vaginally delivered via induction of labor with oxytocin administration after cervical ripening with laminaria. One day after delivery, the platelet count was $948 / \mathrm{mm}^{3}$ (range, 130 to $400 / \mathrm{mm}^{3}$ ). One month after termination of pregnancy, platelets increased to $1,072 / \mathrm{mm}^{3}$ (range, 130 to $400 / \mathrm{mm}^{3}$ ). She is currently being injected INF- $\alpha 2.5$ $\times 10^{6} \mathrm{IU}$ daily for the next pregnancy. As soon as pregnancy is confirmed, aspirin and LMWH are added and the dose is increased, and a platelet apheresis is planned if necessary.

Table 1. Serial measurements of platelet counts, medications, and obstetric events

\begin{tabular}{llll}
\hline Year & Platelet $\left(/ \mathrm{mm}^{3}\right)$ & \multicolumn{1}{c}{ Treatment regimen } & Obstetric event \\
\hline 2005 & 600 & None & Diagnosis \\
2006 & Unknown & None & 1st delivery \\
2008 & 750 & Aspirin $100 \mathrm{mg}$, anagrelide $1 \mathrm{mg}$ & None \\
2010 & 1,663 & Aspirin $100 \mathrm{mg}$, postpartum LMWH & $2 \mathrm{nd}$ delivery \\
2011 & 1,862 & Aspirin $100 \mathrm{mg}$ & Bone marrow biopsy \\
$2011-2013$ & Follow-up loss & None & 1st, 2nd abortion \\
$2013-2015$ & 1,343 & Anagrelide $1 \mathrm{mg}$, aspirin $100 \mathrm{mg}$ & None \\
2015 & 1,291 & INF- $\alpha 5.0 \times 10^{6} \mathrm{IU}$ & 3 rd abortion (9 wk) \\
2016 & 1,478 & INF- $\alpha 2.5 \times 10^{6} \mathrm{IU}$, aspirin $100 \mathrm{mg}$, LMWH $600 \mathrm{IU}$ & 4 th abortion (13 wk) \\
Now & 1,072 & INF- $\alpha 2.5 \times 10^{6} \mathrm{IU}$ & Pregnancy preparation \\
\hline
\end{tabular}

LMWH, low-molecular-weight heparin; INF- $\alpha$, interferon alpha. 
Seo HG, et al. • Recurrent Abortions in Patient with Essential Thrombocythemia

\section{DISCUSSION}

Thrombocythemia is defined as a platelet count exceeding 450/ $\mathrm{mm}^{3}$ observed at least two measurements in one month intervals. Approximately $23 \%$ to $57 \%$ of ET patients carry the JAK2 V617F mutation, but we cannot rule out this disease its absence. This mutation leads to hyperactive JAK signaling, causing the body to make the wrong number of blood cells [7]. Approximately $90 \%$ of patients with ET show an increase in bone marrow cellularity. Megakaryocytic hyperplasia is present. Giant megakaryocytes are frequently observed, and clusters of megakaryocytes may be present. Significant dysplasia of the megakaryocytes is unusual. Hyperplasia of granulocyte and reticulocyte precursors is common. Bone marrow reticulin is usually increased, but collagen fibrosis is uncommon. These patients had no JAK2 V617F mutation, but a bone marrow biopsy was appropriate for diagnosis of this disease. ET is often suspected after a routine blood test shows that a patient has a high platelet count. Other patients may consult their physician after experiencing one or more symptoms of ET. When symptoms are present, they may include fatigue, or may be related to small or large vessel disturbance or bleeding. Many ET patients are asymptomatic. She had no symptoms until she had given birth to two children and during non-pregnancy.

ET is a chronic hematologic malignancy, so it is important for patients to regularly consult with a hematologist and to report any symptoms such as visual disturbances, unexplained pain, numbness, tingling, or bruising. Patients who experience symptoms from ET will require treatment. A small minority of people with ET may later develop acute leukemia (less than $10 \%$ ) or postpolycythemic myelofibrosis (15\%), both of which can be life threatening [8]. To reduce the risk of thrombosis, risk-adapted treatment is recommended. Phlebotomy is the most important and effective treatment modality for patients with erythrocytosis, while cytoreduction using hydroxyurea, busulfan, or INF- $\alpha$ is an ancillary treatment for patients at high risk for thrombosis. Anagrelide is used to decrease platelet counts in patients with thrombocytosis by inhibiting the maturation of platelets from megakaryocytes. Contraception is needed between the last doses of hydroxyurea or anagrelide for 3 to 6 months and planned conception. Interferon (typically pegylated) is sometimes prescribed for ET patients. Women of childbearing age are often treated with interferon because it hasn't been shown to cause birth defects. Platelet reduction with INF- $\alpha$ might be able to further reduce complications of ET during pregnancy and improve fetal outcome. Hydroxycarbamide and anagrelide are contraindicated during pregnancy and nursing. ET can be linked with a three-fold increase in risk of miscarriage. For life-threatening complications, the platelet count can be reduced rapidly using platelet apheresis, a procedure that removes platelets from the blood and returns the remainder to the patient. Nevertheless, further studies with long-term pediatric follow-up are required to assess the safety of INF- $\alpha$ during pregnancy. Therapy with aspirin reduces the risk of spontaneous abortion in the first trimester (19\%-37\%) and the risk of stillbirth (4\%-16\%) when compared with no treatment [9]. Throughout pregnancy, close monitoring of the mother and fetus is recommended. LMWH (e.g., enoxaparin) also may be used. All women are given low-dose aspirin throughout pregnancy and prophylactic LMWH postpartum, whereas LMWH throughout pregnancy and INF- $\alpha$ can be required in patients over 40 years of age with recurrent miscarriage, history of thrombosis, and platelet greater than $1,500 / \mathrm{mm}^{3}$ [10]. However, these treatments did not prevent the recurrent abortions in this patient. Potential new treatments for ET are currently being developed and tested as a result of the discovery of the link between the JAK2 mutation and the incidence of ET. Pegylated interferon, a version of interferon with fewer side effects and easier administration, is being considered as a treatment option for high-risk individuals [11]. Recent trials have shown that ruxolitinib, a JAK inhibitor, has clinical benefits in patients with polycythemia vera who show an inadequate response, or unacceptable side effects, to therapeutic doses of hydroxyurea [12]. When properly monitored and treated, ET patients have an excellent chance of longevity. ET is rare but can occur in women of childbearing age. More research is needed on drugs to prevent early pregnancy miscarriage in ET patients.

\section{REFERENCES}

1. Dinas K, Hatzipantelis E, Karasmanis K, Zepiridis L, Mavromatidis G, Bontis J. Improving the outcome of pregnancy in essential thrombocythaemia: the role of aspirin. J Obstet Gynaecol 2008;28:236-7.

2. Passamonti F, Rumi E, Randi ML, Morra E, Cazzola M. Aspirin in pregnant patients with essential thrombocythemia: a retrospective analysis of 129 pregnancies. J Thromb Haemost 2010;8:411-3.

3. Valera MC, Parant O, Vayssiere C, Arnal JF, Payrastre B. Essential thrombocythemia and pregnancy. Eur J Obstet Gynecol Reprod Biol 2011;158: 141-7.

4. Sohn IS, Kim SN, Lee JY, Lee SJ, Kwon HS, Kim YJ, et al. A case of essential thrombocythemia diagnosed during pregnancy. Korean J Obstet Gynecol 2006;49:2399-403. 
5. Seol YM, Kwon BR, Choi YJ, Lee KW, Shin HJ, Chung JS, et al. The efficacy and adverse effect of hydroxyurea as compared with anagrelide in essential thrombocythemia. Korean J Hematol 2008;43:83-8.

6. Beard J, Hillmen P, Anderson CC, Lewis SM, Pearson TC. Primary thrombocythaemia in pregnancy. Br J Haematol 1991;77:371-4.

7. Cortelazzo S, Finazzi G, Ruggeri M, Vestri O, Galli M, Rodeghiero F, et al. Hydroxyurea for patients with essential thrombocythemia and a high risk of thrombosis. N Engl J Med 1995;332:1132-6.

8. Delage R, Demers C, Cantin G, Roy J. Treatment of essential thrombocythemia during pregnancy with interferon-alpha. Obstet Gynecol 1996;87 (5 Pt 2):814-7.

9. Finazzi G. How to manage essential thrombocythemia. Leukemia
2012;26:875-82.

10. Beauverd Y, Radia D, Cargo C, Knapper S, Drummond M, Pillai A, et al. Pegylated interferon alpha-2a for essential thrombocythemia during pregnancy: outcome and safety: a case series. Haematologica 2016;101: e182-4.

11. Jayasekara WM, Abeyratne SA, Kulathilake C, Gunawardena D, Wijesiriwardena IS. Successful management of a pregnancy complicated by essential thrombocythaemia with pegylated interferon. Ceylon Med J 2015;60:72-3.

12. Kim SY. Advances in treatment for polycythemia vera and essential thrombocythemia. Korean J Med 2016;90:288-92. 